\title{
Panorama da infiltração para ombro doloroso entre especialistas em ombro*
}

\section{Panorama of Infiltration for Painful Shoulder Among Shoulder Specialists}

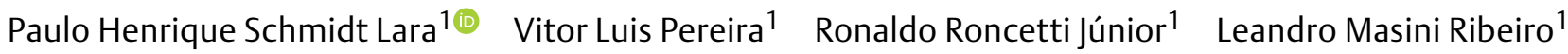 \\ Benno Ejnisman ${ }^{1}$ Paulo Santoro Belangero ${ }^{1}$ \\ ${ }^{1}$ Centro de Traumatologia do Esporte, Departamento de Ortopedia e \\ Traumatologia, Universidade Federal de São Paulo, São Paulo, SP, \\ Brasil \\ Endereço para correspondência Paulo Henrique Schmidt Lara, MD, \\ Rua Estado de Israel, 636, Vila Clementino, São Paulo, SP, \\ São Paulo, 04022-001, Brasil (e-mail: phslara@gmail.com).
}

Rev Bras Ortop 2020;55(1):95-99.

\begin{abstract}
Resumo
Palavras-chave

- ombro

- infiltração

- tendinopatia

- artrose

Objetivo Avaliar como especialistas de ombro têm utilizado a infiltração na sua prática diária.

Métodos Estudo tipo survey interseccional em tempo único, no qual especialistas em ombro e cotovelo responderam a um questionário sobre o uso de infiltrações no ombro doloroso.

Resultados A maior parte (45,9\%) dos entrevistados possui experiência $>10$ anos na área e realizaram até 10 infiltrações nos últimos 12 meses. As principais indicações para infiltração glenoumeral e subacromial são artrose glenoumeral e tendinopatia do manguito rotador, respectivamente. Os portais mais utilizados são o posterior $(52,2 \%)$ para infiltração glenoumeral e o lateral $(57,5 \%)$ na subacromial. A maioria $(75,2 \%)$ dos entrevistados realiza a infiltração ambulatorialmente sem auxílio de métodos de imagem, e a droga mais utilizada é a combinação de corticoide e anestésico. A principal contraindicação citada é a presença de diabetes, e a complicação mais comum é a dor após a infiltração.

Conclusão Infiltrações subacromiais são indicadas especialmente para tratamento de tendinopatias do manguito e bursites, realizadas pelo portal lateral, em ambiente ambulatorial, com baixo índice de complicações em longo prazo. As infiltrações glenoumerais são indicadas especialmente para artrose glenoumeral, com combinação de corticoide e anestésico, realizadas, em sua maioria, ambulatorialmente.
\end{abstract}

Abstract

Objective To assess how shoulder specialists have used infiltration in their daily practice.

Methods A survey study in which shoulder and elbow specialists answered a questionnaire on the use of infiltration in painful shoulders.

\footnotetext{
Trabalho realizado no Centro de Traumatologia do Esporte (CETE), Departamento de Ortopedia e Traumatologia, Universidade Federal de São Paulo (DOT-UNIFESP/EPM), São Paulo, SP, Brasil.
}

recebido

20 de Março de 2018

aceito

02 de Julho de 2018
DOI https://doi.org/

10.1055/s-0039-1697969. ISSN 0102-3616.
Copyright $\odot 2020$ by Sociedade Brasileira License terms de Ortopedia e Traumatologia. Published by Thieme Revinter Publicações Ltda, Rio de Janeiro, Brazil 

Keywords
- shoulder
- infiltration
- tendinopathy
- arthrosis

Results Most of the doctors (45.9\%) have $>10$ years of experience in the area and have carried out up to 10 infiltrations in the last 12 months. The main indications for glenohumeral and subacromial infiltration are glenohumeral arthrosis and rotator cuff tendinopathy, respectively. The most used portals are the posterior $(52.2 \%)$ for glenohumeral infiltration and the lateral $(57.5 \%)$ for subacromial infiltration. The majority of the doctors (752\%) infiltrate in an outpatient setting without imaging methods, and the most commonly used drug is the combination of corticoid and anesthetic. The main contraindication cited is the presence of diabetes, and the most common complication is pain after infiltration.

Conclusion Subacromial infiltrations are indicated especially for the treatment of rotator cuff tendinopathies and bursitis, performed by the lateral portal, in an outpatient setting, with low index of long-term complications. Glenohumeral infiltrations are indicated especially for glenohumeral arthrosis, with a combination of a corticoid and anesthetic, performed mostly in an outpatient setting.

\section{Introdução}

O ombro doloroso é um sintoma frequente no adulto, com uma prevalência de 7 a 34\%, afetando principalmente indivíduos com idade $>40$ anos. ${ }^{1,2}$ Aproximadamente de 10 a $16 \%$ da população geral referem $>1$ episódio de dor no ombro durante a sua vida. ${ }^{1,3,4} \mathrm{~A}$ recuperação pode ser lenta, sendo fatores de mau prognóstico: doentes com idade compreendida entre 45 anos e 54 anos, e quadro doloroso $>3$ meses. ${ }^{1}$ Cerca de 40 a $50 \%$ dos pacientes apresentam dor refratária ao tratamento ou recidiva após 1 ano. ${ }^{5}$

Diversos processos podem originar um quadro clínico de dor no ombro, entre eles alterações inflamatórias dos tendões com ou sem calcificação e doenças degenerativas ósseas ou miotendinosas. ${ }^{4,6}$

A infiltração para melhora dos quadros de dor no ombro tem sido usada há vários anos, sendo que há diversas opções de medicamentos a serem infiltrados, locais de infiltração, auxílio ou não de métodos de imagem e indicações. 0 objetivo do presente estudo é avaliar como especialistas de ombro têm utilizado a infiltração na sua prática diária.

\section{Materias e Métodos}

O presente projeto de pesquisa foi aprovado pelo Comitê de Ética sob o número 70018617.2.0000.5505.

Estudo tipo survey interseccional em tempo único, com uma amostragem não probabilística do tipo conveniência, no qual foram entrevistados 179 ortopedistas especialistas em ombro e cotovelo durante o V Closed Meeting da Sociedade Brasileira de Ombro e Cotovelo em 2017 através de questionário criado pelos autores (-Anexo 1 - disponível online). Desse total, 23 participantes $(12,8 \%)$ referiram não haver realizado nenhuma infiltração nos últimos 12 meses. As respostas seguintes desses indivíduos foram desconsideradas. Havia 250 inscritos e obtivemos uma taxa de resposta de $71,6 \%$. Se considerarmos o total de 890 especialistas, houve uma taxa de resposta de $20,1 \%$. Não houve identificação dos ortopedistas e foi solicitado a eles que cada um respondesse apenas uma vez. Não houve pré-teste para avaliar o questionário.

Os questionários eram autoaplicáveis e foram distribuídos pelos autores do trabalho durante os intervalos dos 3 dias do congresso. Algumas questões permitiam mais de uma resposta, o que levou a uma soma das variáveis acima de $100 \%$.

Foram utilizados os softwares IBM SPSS Statistics for Windows, versão 20.0 (IBM Corp. Armonk, NY, EUA), Minitab 16 (Minitab, LLC, State College, PA, EUA) e Microsoft Excel Office 2010 (Microsoft Corporation, Redmond, WA, EUA) para análise estatística, realizada por meio de teste de igualdade de proporções. Foi definido para este trabalho um nível de significância (valor-p, ou seja, o erro estatístico admitido nas análises foi de $0,05)$. Foi utilizado o teste do qui-quadrado para comparação entre distribuições. Todos os resultados diferentes desse parâmetro serão detalhados. Lembramos também que todos os intervalos de confiança definidos ao longo do trabalho foram assumidos com 95\% de confiança estatística.

\section{Resultados}

Dentre os entrevistados, $46,9 \%$ apresentam $>10$ anos de experiência em cirurgia do ombro; $24 \%$ entre 5 e 10 anos; $19,6 \%$ entre 1 e 5 anos; e 9,5\%, são cirurgiões com $<1$ ano de experiência. Entre a população do estudo, 36,3\% relatam $>10$ indicações de infiltração no último ano. Não existiu diferença estatística entre as respostas de até 10 , e de 10 a 30 infiltrações $(p=0,658)$.

Referente à repetição do procedimento, $39 \%$ indicam apenas após 4 meses, e 5,7\% não insistem em nova infiltração caso o paciente não responda satisfatoriamente à injeção prévia.

As indicações para utilização de infiltração com corticóide subacromial e glenoumeral estão dispostas nas - Tabelas 1 e 2 a seguir.

Quanto ao portal de realização da infiltração subacromial (-Tabela 3), 57,5\% optam pelo acesso lateral, 32,5\% pelo posterior, e $10 \%$ pelo anterior; $4,4 \%$ citaram outros acessos (supraescapular, Neviaser, e superior). Para a infiltração 
Tabela 1 Indicações para infiltração subacromial

\begin{tabular}{|l|l|}
\hline Indicação & $\%$ \\
\hline Tendinopatia do manguito rotador & $55,6 \%$ \\
\hline Bursite & $42,5 \%$ \\
\hline $\begin{array}{l}\text { Lesão parcial do manguito rotador } \\
\text { na face bursal }\end{array}$ & $33,1 \%$ \\
\hline Tendinite calcária & $29,4 \%$ \\
\hline
\end{tabular}

Tabela 2 Indicações para infiltração glenoumeral

\begin{tabular}{|l|l|}
\hline Indicação & $\%$ \\
\hline Artrose glenoumeral & $45,9 \%$ \\
\hline Capsulite adesiva & $32,5 \%$ \\
\hline Sinovite & $21,7 \%$ \\
\hline Tendinopatia do manguito rotador & $8,3 \%$ \\
\hline
\end{tabular}

Tabela 3 Portais de acesso para infiltração subacromial

\begin{tabular}{|l|l|}
\hline Indicação & $\%$ \\
\hline Lateral & $57,5 \%$ \\
\hline Posterior & $32,5 \%$ \\
\hline Anterior & $10 \%$ \\
\hline Outros: supraescapular, Neviaser e superior & $4,4 \%$ \\
\hline
\end{tabular}

Tabela 4 Portais de acesso para infiltração glenoumeral

\begin{tabular}{|l|l|}
\hline Indicação & $\%$ \\
\hline Posterior & $52,2 \%$ \\
\hline Anterior & $35,8 \%$ \\
\hline Outros: supraescapular & $1,3 \%$ \\
\hline Não realizam o procedimento & $11,3 \%$ \\
\hline
\end{tabular}

glenoumeral (-Tabela 4), 52,2\% utilizam ponto de acesso posterior, $35,8 \%$ utilizam o anterior, e $11,3 \%$ não fazem o procedimento. Apenas 2 indivíduos (1,3\%) usam outro acesso (supraescapular).

A Infiltração guiada por ultrassonografia não é utilizada para auxílio no procedimento de infiltração por uma maioria de $88,8 \%$ dos entrevistados, sendo que $10,6 \%$ usam o método para auxílio no acesso glenoumeral, e apenas $0,6 \%$ tanto para o acesso subacromial quanto o glenoumeral; $98,1 \%$ realizam a infiltração subacromial no consultório, e apenas $1,3 \%$ no centro cirúrgico. Quanto à infiltração glenoumeral, 75,2\% infiltram ambulatorialmente, enquanto $13,4 \%$ infiltram no centro cirúrgico.

Quando indagados sobre qual o medicamento escolhido para as infiltrações, $72,3 \%$ dos entrevistados optam pela combinação de corticóide e anestésico; 32\% usam o corticóide de forma isolada, e 8,2\% infiltram apenas anestésico. Somente 0,6\% usam anti-inflamatórios, e 1,9\% usam outras substâncias, como o ácido hialurônico. Quanto ao tipo de corticoide mais usado, 39,4\% optam pela triancinolona, $27,5 \%$ pela dexametasona e $26,9 \%$ pela betametasona.

Quanto às contraindicações ao uso do corticoide, 17,7\% acreditam não haver nenhum tipo de contraindicação, enquanto $82,3 \%$ citaram complicações. Dentre elas, as mais citadas foram diabetes (67,2\%), em especial diabetes mal controlado ou descompensado (7\%), hipertensão arterial (14,7\%), alergias (11,6\%), e glaucoma (10\%).

A principal complicação encontrada pelos entrevistados foi dor após a infiltração (48,1\%), seguida por despigmentação cutânea $(13,5 \%)$ e infecção (3,8\%), enquanto $40,4 \%$ referem não encontrar nenhum tipo de complicação na sua prática. Não encontramos diferença estatística entre a resposta mais recorrente (dor após infiltração) e a resposta nenhuma complicação $(p=0,171)$.

\section{Discussão}

O ombro doloroso é uma condição que pode afetar até entre 26 e $34 \%$ da população adulta. A dor e a consequente disfunção do ombro afetam negativamente a qualidade de vida destes pacientes. Várias doenças cursam com dor no ombro, dentre elas artrose glenoumeral, tendinopatia do manguito rotador, rotura do manguito, capsulite adesiva e tendinite calcária. ${ }^{7}$

A síndrome do manguito rotador é o diagnóstico mais comum nos quadros de ombro doloroso, alcançando prevalência de até $40 \%$, abrangendo desde bursite até roturas completas do manguito, afetando as atividades diárias dos pacientes, especialmente em manobras acima da cabeça. ${ }^{8}$ Caracterizada pela redução do arco de movimento ativo e passivo, além de dor no ombro, a capsulite adesiva aflige $\sim 2 \%$ da população em geral $^{9}$ e até $20 \%$ em diabéticos. ${ }^{10}$ Os depósitos de cálcio no manguito rotador são encontrados em $6,8 \%$ dos pacientes que apresentam ombro doloroso, especialmente alojados no tendão supraespinal. ${ }^{11}$ A osteoartrose glenoumeral é caracterizada por destruição mecânica e biológica da cartilagem articular, sendo condição de difícil controle e altamente debilitante. ${ }^{12}$

A infiltração para o tratamento do ombro doloroso ainda é bastante discutível, porém, observamos que $\sim 83 \%$ dos entrevistados incorporam este método em sua prática, dependendo da doença de base. Randelli et al $^{13}$ conduziram um estudo tipo survey, no qual $72 \%$ dos entrevistados consideraram apropriada ou muito apropriada a infiltração glenoumeral com corticoides como tratamento para capsulite adesiva, e 66\% consideraram apropriada ou muito apropriada a infiltração subacromial para tratamento de casos selecionados de artropatia do manguito.

Dentre as indicações de infiltração subacromial, a mais frequente foi para o tratamento de síndrome do manguito rotador (55,6\% para tendinopatias, $42,5 \%$ para bursite, e $33,1 \%$ para lesões parciais bursais). Segundo Vieira et $\mathrm{al}^{8}{ }^{8}$ os cirurgiões especializados em ombro e cotovelo tendem a optar pelo reparo precoce de lesões completas do manguito rotador, o que pode explicar a baixa incidência de indicação de infiltração para o tratamento destas lesões, apesar de estudos demonstrarem que este procedimento seja capaz de prevenir a necessidade de tratamento cirúrgico em até metade dos casos. ${ }^{14}$ 
Sabe-se que a infiltração com corticoides não é capaz de modificar a história natural da síndrome do manguito rotador, e estudos histológicos em animais mostram mudanças na estrutura molecular do colágeno dos tendões, enfraquecendo-os, após repetidas injeções com corticoide, o que nos leva a crer que essa condição poderia ser traduzida para o ser humano. ${ }^{15,16}$ Entretanto, segundo Bhatia et $\mathrm{al},{ }^{17}$ não existe aumento da incidência de rotura do manguito rotador em pacientes submetidos a múltiplas infiltrações. Assim, acreditamos que este procedimento é uma opção viável no arsenal do tratamento conservador da síndrome do manguito rotador em casos selecionados.

A artrose do ombro foi a principal indicação para infiltração glenoumeral ( $45,9 \%$ ), seguida por capsulite adesiva (33,1\%). Ao comparar estes números com os achados da literatura, nos deparamos com informações divergentes. Por um lado, 31\% dos entrevistados por Randelli et $\mathrm{al}^{13}$ afirmaram que a infiltração glenoumeral é o melhor método de tratamento conservador da capsulite adesiva; porém, apenas $15 \%$ inferem o mesmo sobre sua indicação para artrose do ombro. A infiltração com corticoides para tratamento da artrose glenoumeral encontra pouco suporte na literatura, com estudos com baixa evidência, ${ }^{18}$ a tal ponto que a Academia Americana de Cirurgiões Ortopédicos (AAOS, na sigla em inglês) não indica tal procedimento. ${ }^{19}$ Por outro lado, a utilização deste procedimento no tratamento do ombro congelado apresenta grande suporte, sendo uma opção satisfatória como adjuvante no tratamento conservador. ${ }^{20-22}$

A maioria dos pesquisados opta pelo acesso lateral para infiltração subacromial $(57,5 \%)$ e pelo acesso posterior para a intra-articular $(52,2 \%)$, o que pode ser explicado pela maior presença de estruturas neurovasculares nobres anteriormente, podendo tornar assim a infiltração glenoumeral pela via posterior mais segura. ${ }^{23}$ Este dado se torna importante à luz dos achados de Marder et $\mathrm{al}^{24}{ }^{24}$ que demonstram maior acurácia na infiltração subacromial às cegas pelo portal lateral. Todavia, a familiaridade de cada profissional com a via escolhida certamente é um fator determinante para tal definição.

Poucos profissionais optam por realizar a infiltração subacromial no centro cirúrgico; todavia, a indicação aumenta quando se trata do procedimento intra-articular. Isto pode ser explicado pelo maior desconforto e dor referidos no momento da infiltração glenoumeral. Outro dado importante encontrado é que a maioria dos entrevistados $(88,8 \%)$ não utiliza ultrassonografia para auxiliar no procedimento. A falta de intimidade do cirurgião ortopédico com o aparato, e principalmente, o custo destes aparelhos, dificultam a difusão desta técnica. Todavia, esta prática deve ser repensada, uma vez que infiltrações às cegas apresentam menor acurácia e resultados menos previsíveis. ${ }^{25} \mathrm{Wu}$ et $\mathrm{al}^{26}$ observaram em sua meta-análise que as infiltrações subacromiais guiadas por ultrassom obtiveram melhores resultados no controle da dor causada por síndrome do manguito rotador do que quando realizadas às cegas. Outros estudos demonstram grande variação na acurácia da infiltração glenoumeral se realizada sem auxílio de métodos de imagem (entre 45,7 e $100 \%)^{27,28}$
Dentre as contraindicações, a mais citada foi o diabetes, especialmente quando descompensado. A literatura apresenta poucas evidências de que infiltração subacromial ou glenoumeral possa elevar a glicemia a níveis alarmantes; todavia, é prudente manter vigilância nas semanas seguintes ao procedimento. ${ }^{29}$ Moon et al $^{30}$ estudaram a glicemia após infiltrações glenoumerais com $40 \mathrm{mg}$ de triancinolona em pacientes com e sem diabetes e observaram aumento dos níveis de glicose no primeiro dia após o procedimento e retorno ao parâmetro pré-infiltração em ambos os grupos, sem relato de complicações clinicas.

Por fim, a principal complicação observada pelos pesquisados foi dor após a infiltração, sendo esta uma condição autolimitada; e 40\% dos entrevistados não observaram qualquer efeito adverso.

Os resultados do presente estudo são referentes a uma população especifica de especialistas e não deve ser extrapolado para ortopedistas generalistas. Não houve diferenciação do tipo de infiltração optado para cada doença e, portanto, talvez existam outras nuances não identificadas. Entretanto, o estudo apresenta população bastante representativa, de 170 questionários, em um universo de $\sim 800$ especialistas em ombro e cotovelo em nosso país $(21,25 \%$ da população total de cirurgiões especialistas).

\section{Conclusão}

Dessa forma, conclui-se que o perfil das infiltrações subacromiais é que sua indicação se dá especialmente para tratamento de tendinopatias do manguito e bursites, realizadas pelo portal lateral às cegas em ambiente ambulatorial, com baixo índice de complicações em longo prazo. Já o perfil traçado das infiltrações glenoumerais é que sua utilização se dá principalmente para artrose glenoumeral, com combinação de corticoide e anestésico, realizada por via posterior às cegas e, em sua maioria, ambulatorialmente.

Conflito de Interesses

Os autores declaram não haver conflito de interesses.

\section{Referências}

1 Diercks R, Bron C, Dorrestijn O, et al; Dutch Orthopaedic Association. Guideline for diagnosis and treatment of subacromial pain syndrome: a multidisciplinary review by the Dutch Orthopaedic Association. Acta Orthop 2014;85(03):314-322

2 Codsi MJ. The painful shoulder: when to inject and when to refer. Cleve Clin J Med 2007;74(07):473-474, 477-478, 480-482 passim

3 Stevenson K. Evidence-based review of shoulder pain. Musculoskelet Care 2006;4(04):233-239

4 Holt TA, Mant D, Carr A, et al. Corticosteroid injection for shoulder pain: single-blind randomized pilot trial in primary care. Trials 2013;14:425

5 Laslett M, Steele M, Hing W, McNair P, Cadogan A. Shoulder pain patients in primary care-part 1: Clinical outcomes over 12 months following standardized diagnostic workup, corticosteroid injections, and community-based care. J Rehabil Med 2014; 46(09):898-907

6 Saccomanni B. Inflammation and shoulder pain-a perspective on rotator cuff disease, adhesive capsulitis, and osteoarthritis: conservative treatment. Clin Rheumatol 2009;28(05):495-500 
7 Sun Y, Chen J, Li H, Jiang J, Chen S. Steroid Injection and Nonsteroidal Anti-inflammatory Agents for Shoulder Pain: A PRISMA Systematic Review and Meta-Analysis of Randomized Controlled Trials. Medicine (Baltimore) 2015;94(50):e2216

8 Vieira FA, Olawa PJ, Belangero PS, Arliani GG, Figueiredo EA, Ejnisman B. Rotator cuff injuries: current perspectives and trends for treatment and rehabilitation. Rev Bras Ortop 2015;50(06):647-651

9 Manske RC, Prohaska D. Diagnosis and management of adhesive capsulitis. Curr Rev Musculoskelet Med 2008;1(3-4):180-189

10 Dehghan A, Pishgooei N, Salami MA, et al. Comparison between NSAID and intra-articular corticosteroid injection in frozen shoulder of diabetic patients; a randomized clinical trial. Exp Clin Endocrinol Diabetes 2013;121(02):75-79

11 Diehl P, Gerdesmeyer L, Gollwitzer H, Sauer W, Tischer T. Calcific tendinitis of the shoulder. Orthopade 2011;40(08):733-746

12 Millett PJ, Gobezie R, Boykin RE. Shoulder osteoarthritis: diagnosis and management. Am Fam Physician 2008;78(05):605-611

13 Randelli P, Arrigoni P, Cabitza F, Ragone V, Cabitza P. Current practice in shoulder pathology: results of a web-based survey among a community of 1,084 orthopedic surgeons. Knee Surg Sports Traumatol Arthrosc 2012;20(05):803-815

14 Plafki C, Steffen R, Willburger RE, Wittenberg RH. Local anaesthetic injection with and without corticosteroids for subacromial impingement syndrome. Int Orthop 2000;24(01):40-42

15 Lee HJ, Kim YS, Ok JH, Lee YK, Ha MY. Effect of a single subacromial prednisolone injection in acute rotator cuff tears in a rat model. Knee Surg Sports Traumatol Arthrosc 2015;23(02):555-561

16 Wei AS, Callaci JJ, Juknelis D, et al. The effect of corticosteroid on collagen expression in injured rotator cuff tendon. J Bone Joint Surg Am 2006;88(06):1331-1338

17 Bhatia M, Singh B, Nicolaou N, Ravikumar KJ. Correlation between rotator cuff tears and repeated subacromial steroid injections: a case-controlled study. Ann R Coll Surg Engl 2009;91(05):414-416

18 Colen S, Geervliet P, Haverkamp D, Van Den Bekerom MP. Intraarticular infiltration therapy for patients with glenohumeral osteoarthritis: A systematic review of the literature. Int J Shoulder Surg 2014;8(04):114-121

19 Izquierdo R, Voloshin I, Edwards S, et al. American academy of orthopaedic surgeons clinical practice guideline on: the treat- ment of glenohumeral joint osteoarthritis. J Bone Joint Surg Am 2011;93(02):203-205

20 Bal A, Eksioglu E, Gulec B, Aydog E, Gurcay E, Cakci A. Effectiveness of corticosteroid injection in adhesive capsulitis. Clin Rehabil 2008;22(06):503-512

21 De Carli A, Vadalà A, Perugia D, et al. Shoulder adhesive capsulitis: manipulation and arthroscopic arthrolysis or intra-articular steroid injections? Int Orthop 2012;36(01):101-106

22 Wang W, Shi M, Zhou C, et al. Effectiveness of corticosteroid injections in adhesive capsulitis of shoulder: A meta-analysis. Medicine (Baltimore) 2017;96(28):e7529

23 Bell AD, Conaway D. Corticosteroid injections for painful shoulders. Int J Clin Pract 2005;59(10):1178-1186

24 Marder RA, Kim SH, Labson JD, Hunter JC. Injection of the subacromial bursa in patients with rotator cuff syndrome: a prospective, randomized study comparing the effectiveness of different routes. J Bone Joint Surg Am 2012;94(16):1442-1447

25 Henkus HE, Cobben LP, Coerkamp EG, Nelissen RG, van Arkel ER. The accuracy of subacromial injections: a prospective randomized magnetic resonance imaging study. Arthroscopy 2006;22 (03):277-282

$26 \mathrm{Wu}$ T, Song HX, Dong Y, Li JH. Ultrasound-guided versus blind subacromial-subdeltoid bursa injection in adults with shoulder pain: A systematic review and meta-analysis. Semin Arthritis Rheum 2015;45(03):374-378

27 Tobola A, Cook C, Cassas KJ, et al. Accuracy of glenohumeral joint injections: comparing approach and experience of provider. J Shoulder Elbow Surg 2011;20(07):1147-1154

28 Kraeutler MJ, Cohen SB, Ciccotti MG, Dodson CC. Accuracy of intra-articular injections of the glenohumeral joint through an anterior approach: arthroscopic correlation. J Shoulder Elbow Surg 2012;21(03):380-383

29 Povlsen B, Povlsen SD. Steroid injection for shoulder pain causes prolonged increased glucose level in type 1 diabetics. BMJ Case Rep 2014;2014:bcr2014203777

30 Moon HJ, Choi KH, Lee SI, Lee OJ, Shin JW, Kim TW. Changes in blood glucose and cortisol levels after epidural or shoulder intraarticular glucocorticoid injections in diabetic or nondiabetic patients. Am J Phys Med Rehabil 2014;93(05):372-378 